\title{
INFORMACIONES ECOLÓGICAS SOBRE COPROPHANAEUS (COPROPHANAEUS) GAMEZI ARNAUD (COLEOPTERA: SCARABAEINAE: PHANAEINI) EN UN SISTEMA AGROPASTORIL EN LA DEPRESIÓN DE MARACAIBO, ESTADO ZULIA, VENEZUELA
}

\author{
Jorge GÁMEZ y Raffaele ACCONCIA \\ Fundación Entomológica Andina, Mérida, VENEZUELA. \\ e-mail: funeave2008@gmail.com
}

Gámez, J. y R. Acconcia. 2009. Informaciones ecológicas sobre Coprophanaeus (Coprophanaeus) gamezi Arnaud (Coleoptera: Scarabaeidae: Phanaeini) en un sistema agropastoril en la depresión de Maracaibo, Estado de Zulia, Venezuela. Acta Zoológica Mexicana (n. s.), 25(2): 387-396.

RESUMEN. Este trabajo reporta los resultados de campo sobre la biología de Coprophanaeus (Coprophanaeus) gamezi Arnaud realizados tanto en un pastizal introducido como en un cultivo de plátano. Para ello, se instalaron necrotrampas para evaluar su actividad anual, diaria y el uso del espacio. Se colectó todo el año concentrándose las mayores abundancias durante los meses de máximas precipitaciones. Esta especie utiliza los dos hábitats evaluados colectándose en mayor abundancia, en el cultivo de plátano. Presentó actividad diaria bimodal exclusivamente en el crepúsculo con una inversión de tiempo de aproximadamente 60 minutos en 24 horas y con reinicio de la función exploratoria cada 13 horas. Manifiesta actividad diaria preferencial, en los hábitats evaluados, por intensidades de luz baja (ocaso: 0 - 30 Lux; alba: 0 - 3 Lux), humedad atmosférica alta (entre $87.6 \%$ y $89 \%$ para el ocaso y entre $95.3 \%$ y $96.7 \%$ para el alba), temperaturas entre $27.3^{\circ} \mathrm{C}$ y $27.7^{\circ} \mathrm{C}$ para el ocaso y $24.9^{\circ} \mathrm{C}$ y $25.1^{\circ} \mathrm{C}$ para el alba. Se destaca que el $73 \%$ de las colectas fueron registradas en el ocaso, posiblemente como una consecuencia de interacciones entre el ciclo endógeno particular de la especie y ciertos factores ambientales, principalmente, la temperatura e intensidad de la luz. Coprophanaeus (C.) gamezi es una de las especies necrófagas más importantes en los hábitats abiertos o semiabiertos analizados y su conspicua presencia y distribución se ha favorecido debido a la desaparición de la selva en la Depresión de Maracaibo.

Palabras Clave: Coleóptero necrófago, Scarabaeinae, actividad anual, actividad diaria, uso del hábitat, deforestación.

Gámez, J. \& R. Acconcia. 2009. Ecological informations on Coprophanaeus (Coprophanaeus) gamezi Arnaud (Coleoptera: Scarabaeidae: Phanaeini) in a rural agricultural area in the depression of Maracaibo, Zulia State, Venezuela. Acta Zoológica Mexicana (n. s.), 25(2): 387-396.

ABSTRACT. This paper reports the results of fields studies on the biology of Coprophanaeus (C.) gamezi Arnaud conducted in induced pastureland and banana plantations in the Lake Maracaibo Depression of the state of Zulia, Venezuela. Carrion - baited pitfall traps were used to monitor annual and daily activity cycles. Greatest abundance was observed in the banana plantations. Daily activity

Recibido: 13/10/2008; aceptado: 12/05/2009. 
cycle was crepuscular and bimodal with approximately 30 min periods of activity at dusk and dawn. Daily rhythm was marked by a preference for low light intensities ( 0 - 30 Lux sunset, $0-3$ Lux sunrise), higher humidities $\left(87.6 \%-89 \%\right.$ sunset; $95.3 \%-96.7 \%$ sunrise), and temperatures between $27.3^{\circ} \mathrm{C}$ and $27.7^{\circ} \mathrm{C}$ sunset and $24.9^{\circ} \mathrm{C}$ and $25.1{ }^{\circ} \mathrm{C}$ sunrise. Most $(73 \%)$ of the captured specimens were collected at sunset, perhaps as a consequence of interactions between an endogenous rhythm and certain environmental variables, especially temperature and light intensity. Coprophanaeus gamezi is one of the most important necrophages in open and semi open habitats studied where rainforest has been cut in the Maracaibo Depression.

Key Words: Necrophagous Coleoptera, Scarabaeinae, annual and daily cycles, habitat utilization, deforestation.

\section{INTRODUCCIÓN}

Dentro de los ambientes fisiográficos de Venezuela, hacia el occidente del país, se encuentran las regiones de Montañas con la subregión Cordillera de Los Andes y la de Llanuras Bajas con las subregiones Llanos y Depresión de Maracaibo (Huber 1997). En esta última, se ha registrado a dos especialistas necrófagos pertenecientes a la subtribu Phanaeini: Coprophanaeus (Coprophanaeus) telamon nevinsoni Arnaud \& Gámez y Coprophanaeus (Coprophanaeus) gamezi Arnaud. Las observaciones sobre necrófagos Phanaeini del género Coprophanaeus en Venezuela, reflejan la preferencia de las mismas por ambientes selváticos o aquellos derivados, incluso cultivos, que conserven cierta estructuración vertical, es decir, que mantengan elementos de los estratos arbóreo y arbustivo y en donde los corredores de vegetación promuevan la conexión entre los hábitats (Gámez 2004, Gámez et al. 2006). Coprophanaeus telamon nevinsoni se asocia con las condiciones antes descritas, en cambio Coprophanaeus gamezi sólo ha sido colectada en pastizales (citado como Coprophanaeus jasius (Olivier) en Gámez 2004) y se han detectado poblaciones bien establecidas en la Depresión de Maracaibo. En este trabajo se analizan los siguientes aspectos de la ecología y comportamiento de Coprophanaeus. (C.) gamezi:

Determinar la actividad anual y diaria de la especie.

Evaluar la preferencia de los escarabajos por dos hábitats característicos de un sistema agropastoril: pastizal introducido y cultivo de plátano.

Determinar los valores funcionales de la especie (Valores Indicadores y Tolerancias), en los hábitats característicos de la Depresión de Maracaibo, relacionando las abundancias mensuales con algunas variables ambientales.

\section{MATERIALES Y MÉTODOS}

Área de estudio: Se ubicó el estudio dentro de la Depresión de Maracaibo en la finca El Recreo, sector Bancada de Limones, Municipio Colón del estado Zulia entre las coordenadas UTM 202998E y 977272 N (Fig. 1) a una altitud de 180 m s.n.m. En la zona se presenta una temperatura media anual de $26.7^{\circ} \mathrm{C}$ y una precipitación anual de $1785 \mathrm{~mm}$ con dos periodos máximos que ocurren en los meses de abril y mayo 


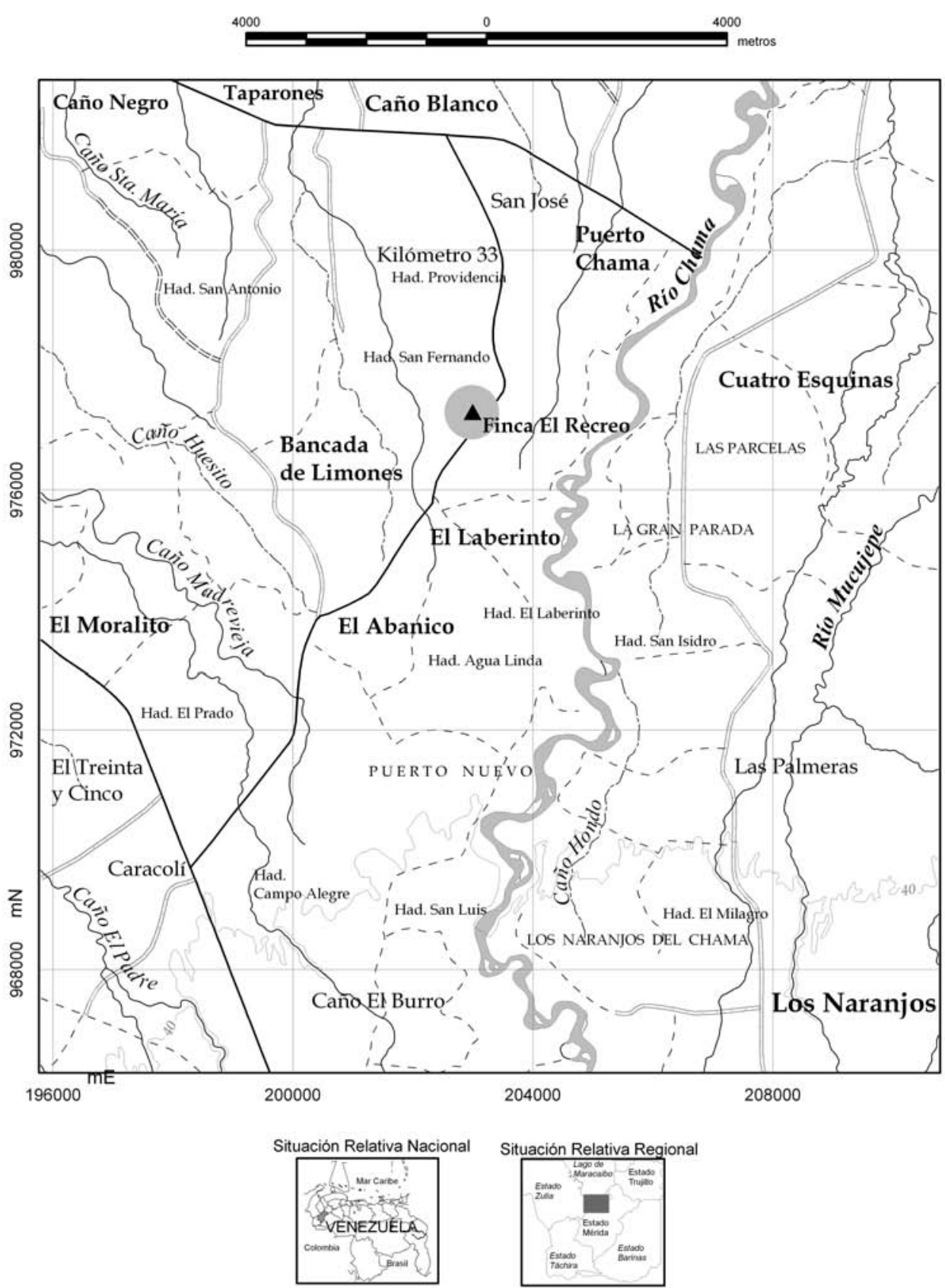

Figura 1. Ubicación del área de estudio $\triangle$ sector Bancada de Limones Municipio Colón, Estado Zulia, Venezuela. 
para el primero y octubre y noviembre para el segundo (Ministerio del Ambiente y de los Recursos Naturales Renovables. Sistema Nacional de Información Hidrológica y Meteorológica. 2007). La unidad de producción agropecuaria se ubica en suelo aluvial de reciente formación con textura arenosa. Allí se concentran 51 ha de pasto introducido (Cynodon plectostachyum Pilger, comúnmente llamado pasto estrella) permitiendo el pastoreo de ganado para la producción de carne y aproximadamente 27 ha de plátano (Musa. AAB.c.v.Harton). A través del año hubo asistencia permanente de éste cultivo para impedir el crecimiento de plantas herbáceas, sin embargo, y fundamentalmente en los caminos de acceso a la platanera, se pudo registrar a representantes de las familias Gramineae, Euphorbiaceae, Leguminoceae, Commelinaceae y Malvaceae.

Diseño del muestreo: Para la determinación del patrón de actividad anual y preferencia de hábitat se efectuaron muestreos, una vez por mes, de enero a diciembre de 2007 utilizándose cinco necrotrampas de caída en el pastizal e igual número en el cultivo de plátano. Las mismas se instalaron en el suelo, dispuestas en transectos con $20 \mathrm{~m}$ de separación entre sí. El modelo de trampa es descrito en Gámez et al. (2006). Como cebo se utilizó pescado en descomposición y como líquido conservante, contenido dentro del envase colector, agua saturada con cloruro de sodio. Las trampas al instalarse permanecieron activas por 14 horas desde las 17:00 horas hasta las 08:00 horas del día siguiente. Al final de dicho periodo se colaba el contenido de las mismas depositándose luego los especímenes en envases de traslado. Posteriormente, todo el material, por hábitat y por periodo de actividad se lavó con agua corriente, se separaron las diferentes especies y se preservaron en alcohol isopropílico al $70 \%$ en frascos debidamente etiquetados. Coprophanaeus $(C$.) gamezi fue procesada para determinar el largo total de la especie, la relación porcentual entre machos y hembras, las proporciones en función de los hábitats evaluados y las abundancias mensuales.

El análisis de la actividad diaria se efectúo tanto en el pastizal como en el platanal utilizándose las necrotrampas de muestreos mensuales con revisiones permanentes desde las 17:00 hasta las 08:00 horas del siguiente día. Para recabar esta información se efectuaron los registros alternando un mes en el pastizal y otro en el platanal. En cada uno, se emplearon a dos observadores y a un registrador de datos. Los observadores transmitían, a este último y por radio, información sobre la presencia de los escarabajos en las trampas, en vuelo, caminando y otros aspectos comportamentales a través de observaciones permanentes en las trampas y áreas circundantes. El registrador asentaba la información y, a su vez, registraba los valores de temperatura ambiental $\left({ }^{\circ} \mathrm{C}\right)$, humedad atmosférica $(\%)$ e intensidad de la luz (Lux). Con estos registros ambientales, ponderándolos por las abundancias mensuales de la especie, se calcularon los Valores Indicadores (VIi) y las tolerancias (ti) -rangos- obteniéndose respuestas estimadas de la especie a las variables 
consideradas en los hábitats estudiados (Fariñas 1996). A tal efecto, se utilizaron las siguientes ecuaciones:

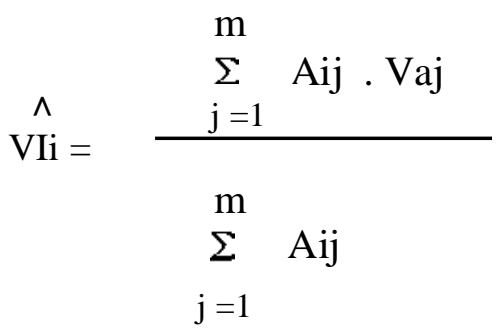

Donde:

$\mathrm{VIi}=$ Valor indicador de la especie

Aij = Abundancia de la especie $\mathrm{i}$ en los hábitats evaluados $\mathrm{j}$

Vaj $=$ Valor de variable ambiental en los hábitats evaluados $\mathrm{j}$

$\mathrm{m}=$ Número de hábitats observados

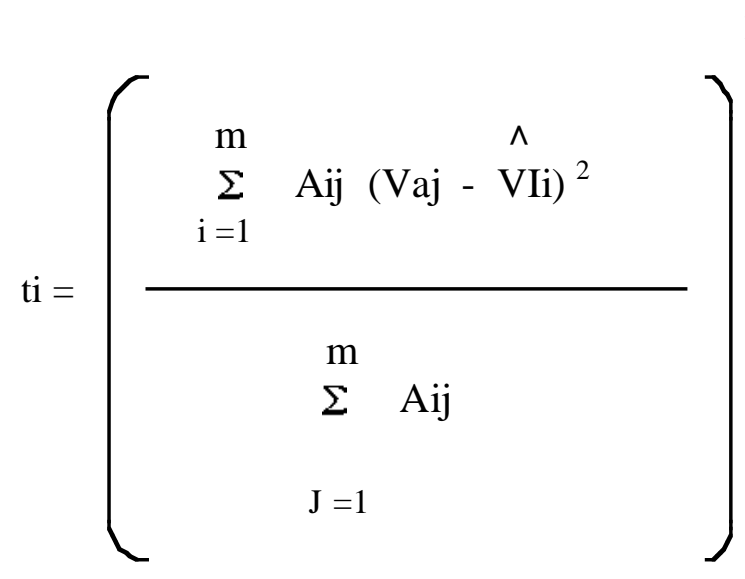

Donde,

$\mathrm{ti}=$ Tolerancia de la especie

Aij = Abundancia de la especie

Vaj $=$ Valor de la variable ambiental

VIi $=$ Valor indicador estimado

$\mathrm{m}=$ Número de hábitats observados

Con el fin de verificar y complementar las observaciones sobre la preferencia de la especie por los hábitats presentes en la Depresión de Maracaibo, se instalaron 
necrotrampas siguiéndose un transecto y con 20 m de separación entre si: tres dentro de un manchón selvático, tres en el ecotono selva - pastizal introducido y tres en el pastizal. El manchón selvático de aproximadamente 27 ha, se ubica dentro de la hacienda El Amparo en el Km 47 (Municipio Alberto Adriani del estado Mérida) al margen izquierdo de la vía El Vigía - Santa Bárbara del Zulia realizándose los registros por 48 horas consecutivas (13-05-08 al 15-05-08).

\section{RESULTADOS}

Actividad anual y uso del espacio. Coprophanaeus (C.) gamezi fue colectada durante todo el año concentrándose las mayores abundancias durante los meses de máximas precipitaciones (Fig. 2). Del total de las capturas, el 56\% correspondió a los machos.

Con relación a la preferencia de la especie por los hábitats evaluados, el 54\% de los individuos se concentraron en el platanal y el restante $46 \%$ en el pastizal introducido. En función de las observaciones realizadas en el manchón selvático, se confirma la ausencia de Coprophanaeus (C.) gamezi en el ecotono y dentro del mismo, allí se colectó a Coprophanaeus telamon nevinsoni, la otra especie carroñera de la tribu Phanaeini presente en la Depresión de Maracaibo (Gámez 2004).

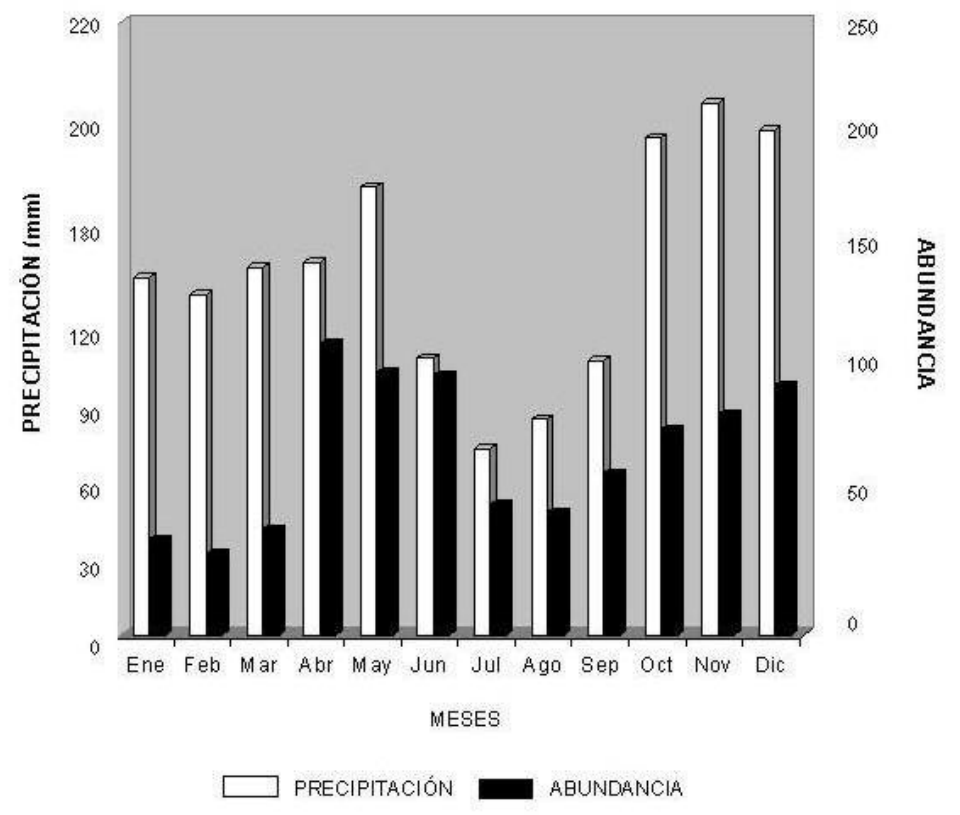

Figura 2. Abundancia de Coprophanaeus gamezi relacionada con el régimen de precipitación. 
Actividad diaria. Con base en los registros y colectas efectuadas, Coprophanaeus (C.) gamezi presentó actividad diaria bimodal, coincidiendo con lo observado para otras especies (Cuadro 1). En los hábitats estudiados la especie presenta en promedio 27 minutos de actividad en el alba y 29 minutos en el ocaso lo que representa una inversión neta de tiempo, en inspección de vuelo, de aproximadamente 60 minutos en 24 horas y en función de la condición crepuscular exhibida, inicia actividad cada 13 horas. Los Valores Indicadores reflejados por la especie en estos intervalos de tiempo se concentran en el cuadro 2. Con relación a los rangos de tolerancia, dentro de los lapsos de tiempo de actividad de la especie y en función de los factores ambientales considerados, se llegó a la conclusión de que Coprophanaeus (C.) gamezi presenta actividad diaria preferentemente con intensidades de luz baja (ocaso: 0 y 30 Lux; alba: 0 y 3 Lux), humedad atmosférica alta (entre $87.6 \%$ y $89 \%$ para el ocaso y entre $95.3 \%$ y $96.7 \%$ para el alba), y temperaturas entre $27.3^{\circ} \mathrm{C}$ y $27.7^{\circ} \mathrm{C}$ para el ocaso y $24.9^{\circ} \mathrm{C}$ y $25.1^{\circ} \mathrm{C}$ para el alba. Se destaca, que en función de las recolecciones efectuadas, tanto en el alba como en el ocaso, el $73 \%$ de las capturas ocurrieron en éste último periodo de tiempo.

\section{DISCUSIÓN}

Con la información recabada para Coprophanaeus gamezi, se confirma la condición crepuscular exhibida por algunas de las especies del género Coprophanaeus estudiadas (Cuadro 1). Dentro de este ciclo diario se destacan, de igual forma, lapsos de tiempo similares empleados en el inicio y cierre de las actividades dentro de una aparente corta acción tanto en alba como en el ocaso, en las funciones de búsqueda del recurso carroña. En este sentido Gill (1991), refiriéndose específicamente al género Coprophanaeus, ha conjeturado que en función de la menor disponibilidad y predictibilidad de la carroña, en comparación con el estiércol, un periodo de vuelo corto a alta velocidad podría ser suficiente para determinar si la carroña está localmente disponible promoviéndose, de igual modo, ahorro energético.

En la Depresión de Maracaibo se encuentran activas en prácticamente el mismo intervalo de tiempo a Coprophanaeus telamon nevinsoni y Coprophanaeus $(C$.) gamezi. Son de similares tamaños (C. t. nevinsoni: $24 \mathrm{~mm}$; C. (C.) gamezi: $26 \mathrm{~mm}$ ) y la coexistencia, como se ha señalado, se da en función de que Coprophanaeus telamon nevinsoni se asocia con hábitats selváticos o modificados y Coprophanaeus gamezi corresponde a una especie de hábitats abiertos (pastizales introducidos o sabanas) o semiabiertos como el platanal. Se establece claramente que si la selva en la Depresión de Maracaibo no hubiese desaparecido en la magnitud en la que ha sido sometida, Coprophanaeus (C.) gamezi y otras especies presentes tales como Digitonthophagus gazella (Fabricius) no se observarían en dicha región. AL respecto sería importante investigar, por ejemplo, si Coprophanaeus $(C)$ gamezi ha incrementado su rango de distribución por la antropización del paisaje utilizando 
Gámez y Acconcia: Informaciones ecológicas sobre Coprophanaeus gamezi en Venezuela

Cuadro 1. Lapsos de tiempo invertido por algunas especies del género Coprophanaeus en actividad diaria de vuelo exploratorio.

\begin{tabular}{|c|c|c|c|}
\hline Especies & Alba & Ocaso & Fuente \\
\hline Coprophanaeus gamezi Arnaud & $\begin{array}{l}\text { 06:09 - 06:41 horas } \\
\text { (Pastizal - Platanal) }\end{array}$ & $\begin{array}{l}\text { 19:00 - 19:38 horas } \\
\text { (Pastizal - Platanal) }\end{array}$ & Presente Investigación \\
\hline \multirow[t]{2}{*}{$\begin{array}{l}\text { Coprophanaeus tel } \\
\text { Arnaud \&Gámez }\end{array}$} & $\begin{array}{c}\text { 06:00 - 06:45 horas } \\
\text { (Selva) }\end{array}$ & $\begin{array}{c}\text { 06:00 }-06: 25 \text { horas } \\
\text { (Cultivo) }\end{array}$ & Gámez et al. 2006 \\
\hline & $\begin{array}{c}\text { 18:30 }-19: 30 \text { horas } \\
\text { (Selva) }\end{array}$ & $\begin{array}{c}\text { 18:40 }-19: 15 \text { horas } \\
\text { (Cultivo) }\end{array}$ & \\
\hline $\begin{array}{l}\text { Coprophanaeus telamon telamon } \\
\text { (Harold) }\end{array}$ & $\begin{array}{c}\text { 06:02 - 06:31 horas } \\
\text { (Selva) }\end{array}$ & $\begin{array}{c}\text { 19:03 - 19:40 horas } \\
\text { (Selva) }\end{array}$ & $\begin{array}{l}\text { Jorge Gámez, observaciones } \\
\text { personales: Sierra de San Luis, } \\
\text { Estado Falcón. Venezuela. }\end{array}$ \\
\hline $\begin{array}{l}\text { Coprophanaeus christophorowi } \\
\text { (Olsoufieff) }\end{array}$ & $\begin{array}{c}\text { 06:00 - 07:00 horas } \\
\text { (Selva) }\end{array}$ & $\begin{array}{c}\text { 18:00 }-19: 00 \text { horas } \\
\text { (Selva) }\end{array}$ & $\begin{array}{l}\text { Feer \& Pincebourde } \\
2005\end{array}$ \\
\hline $\begin{array}{l}\text { Coprophanaeus parvulus } \\
\text { (Olsoufieff) }\end{array}$ & $\begin{array}{c}\text { 06:00 - 07:00 horas } \\
\text { (Selva) }\end{array}$ & $\begin{array}{c}\text { 18:00 - 19:00 horas } \\
\text { (Selva) }\end{array}$ & $\begin{array}{l}\text { Feer \& Pincebourde } \\
2005\end{array}$ \\
\hline $\begin{array}{l}\text { Coprophanaeus jasius } \\
\text { (Olivier) }\end{array}$ & $\begin{array}{c}\text { 06:00 - 07:00 horas } \\
\text { (Selva) }\end{array}$ & $\begin{array}{c}\text { 18:00 - 19:00 horas } \\
\text { (Selva) }\end{array}$ & $\begin{array}{l}\text { Feer \& Pincebourde } \\
2005\end{array}$ \\
\hline $\begin{array}{l}\text { Coprophanaeus dardanus } \\
\text { (Macleay) }\end{array}$ & $\begin{array}{c}\text { 06:00 - 07:00 horas } \\
\text { (Selva) }\end{array}$ & $\begin{array}{c}\text { 18:00 - 19:00 horas } \\
\text { (Selva) }\end{array}$ & $\begin{array}{l}\text { Feer \& Pincebourde } \\
2005\end{array}$ \\
\hline $\begin{array}{l}\text { Coprophanaeus lancifer } \\
\text { (Linnaeus) }\end{array}$ & & $\begin{array}{c}\text { 18:00 - 19:00 horas } \\
\text { (Selva) }\end{array}$ & $\begin{array}{l}\text { Pincebourde } 2002 \\
\text { Feer \& Pincebourde } 2005\end{array}$ \\
\hline
\end{tabular}

algún corredor ecológico. Esto por el hecho de que está registrada para los Llanos de Venezuela y desde esta zona a la Depresión de Maracaibo media la cordillera de Los Andes venezolanos.

Ahora bien, $73 \%$ de los individuos se colectaron en el ocaso lo que coincide con las observaciones existentes para Coprophanaeus telamon nevinsoni (Gámez et al. 2006). Al parecer, en el ocaso la especie conseguiría un mejor desempeño. En este 
sentido se considera que la actividad estacional y diaria pudiera estar relacionada con la biología térmica de los escarabajos permitiendo la segregación de las especies o la coexistencia de aquellas con similares requerimientos ecológicos, entonces, se ha propuesto el concepto de nicho térmico para explicar las interacciones interespecíficas y la distribución de determinadas especies (Verdú et al. 2004, Verdú et al. 2006, Verdú et al. 2007). Otras investigaciones, enfatizan la importancia de un ciclo endógeno para las especies modulable, por los factores ambientales en donde la intensidad de la luz pareciera ser preponderante (Wensler 1974, Mena et al. 1989, Houston \& McIntyre 1985, Martin - Piera et al. 1994). Para el caso de Coprophanaeus gamezi, siendo una especie paracóprida que permanece en las galerías, se reactivaría en función de su ciclo endógeno característico pudiendo la especie conseguir una eficiente autorregulación de la temperatura hacia el ocaso impulsando su patrón de acción para las actividades de exploración que realiza bajo intensidades de luz características. Hacia el alba es probable que una disminución de más de dos grados centígrados en la temperatura ambiental limite la actividad de los escarabajos aunque la misma se desarrolle en intensidades de luz similares a las del ocaso (Cuadro 2).

Cuadro 2. Valores Indicadores de las preferencias de Coprophanaeus gamezi Arnaud para algunos parámetros ambientales en la Depresión de Maracaibo.

\begin{tabular}{lccc}
\hline & $\begin{array}{c}\text { Intensidad de la Luz } \\
(\text { Lux })\end{array}$ & $\begin{array}{c}\text { Temperatura Ambiental } \\
\left({ }^{\circ} \mathbf{C}\right)\end{array}$ & $\begin{array}{c}\text { Humedad Atmosférica } \\
\%\end{array}$ \\
\hline Alba & 1 & 25 & 96 \\
Ocaso & 1 & 27.5 & 88 \\
\hline
\end{tabular}

AGRADECIMIENTOS. A W. D. Edmonds, quien revisó una versión preliminar dando sugerencias importantes para la presentación definitiva del manuscrito, siempre ha colaborado asesorándonos técnicamente y proporcionándonos la literatura que se ha necesitado. El documento también se benefició de los comentarios editoriales y de dos árbitros anónimos. La familia Ramírez, en especial, Onésimo, José y Nereida Ramírez proporcionaron todas las facilidades para la realización de los trabajos de campo en la finca El Recreo. De igual manera, el señor Teodoro Zerpa permitió expeditamente el trabajo de campo complementario en la hacienda El Amparo. José R.Verdú y Lucrecia Arellano, facilitaron bibliografía esencial. Angélica Gámez desarrolló los cuadros que se presentan y colaboró en la transcripción del manuscrito. 


\section{LITERATURA CITADA}

Feer, F. \& S. Pincebourde. 2005. Diel flight activity and ecological segregation within an assemblage of tropical forest dung and carrion beetles. Journal of Tropical Ecology. 21: 21-30.

Gámez, J. 2004. Phanaeini (Coleoptera: Scarabaeinae) de la cordillera de Los Andes, Depresión de Maracaibo y Llanos de Venezuela. Memoria Fundación La Salle de Ciencias Naturales. 158: 43-60.

Gámez, J; E. Mora y R. Acconcia. 2006. Informaciones ecológicas sobre Coprophanaeus (Coprophanaeus) telamon nevinsoni Arnaud \& Gámez (Coleoptera: Scarabaeinae: Phanaeini) en un sector de selva húmeda submontana en Mérida, Venezuela. Acta Zoológica Mexicana. (n.s). 22 (3): 95-105.

Gill, B. 1991. Dung Beetle in Tropical American Forest. Pp. 211-229. In: I. Hanski \& Y. Cambefort (eds). Dung Beetle Ecology. Princeton University Press, New Jersey.

Houston, W. W. \& P. McIntyre. 1985. The daily onset of flight in the crepuscular dung beetle Onitis alexis. Entomologia Experimentalis et Applicata. 39: 223-232.

Huber, O. 1997. Ambientes fisiográficos y vegetales de Venezuela. Pp. 279-298. En. E. La Marca (ed). Vertebrados Actuales y Fósiles de Venezuela. Serie Catálogo Zoológico de Venezuela, Vol. 1. Museo de Ciencia y Tecnología de Mérida.

Martin - Piera, F.; I. Sanmartín \& J. M. Lobo. 1994. Observaciones sobre el ritmo de actividad diaria en escarabeidos telecópridos (Coleoptera, Scarabaeidae). Bulletin de la Société Entomologique de France. 99 (5): 463-470.

Mena, J; E. Galante \& C. Lumbreras. 1989. Daily flight activity of Scarabaeidae and Geotrupidae (Col.) and analysis of the factors determinig this activity. Ecología Mediterránea. 15 (1/2): 69-80.

Pincebourde, S. 2002. Selection de l'habitat luminaux chez des bousiers Phanaeini (Coleoptera, Scarabaeidae) de forêt primaire en Guyane française. Thesis. Université Francois Rabelais, Tours, France.

Verdú, J. R.; L. Arellano \& C. Numa. 2006. Thermoregulation in endothermic dung beetle (Coleoptera: Scarabaeidae): Effect of body size and ecophysiological constraints in flight. Journal of Insect Physiology. 52: 854-860.

Verdú, J. R; L. Arellano; C. Numa \& E. Micó. 2007. Roles of endothermy in niche differentiation for ball - rolling dung beteles (Coleoptera: Scarabaeidae) along an altitudinal gradient. Ecological Entomology. 32: 544-551.

Verdú, J. R; A. Díaz \& E. Galante. 2004. Thermoregulatory strategies in two closely related sympatric Scarabaeus species (Coleoptera: Scarabaeinae). Physiological Entomology. 29: 32-38.

Wensler, R. J. 1974. Crepuscular activity of adult Seriscesthis germinata (Coleoptera: Scarabaeidae): influence of circadian rhythmicity and light intensity. New Zealand Journal of Zoology. 1 (2): 197-204. 